\title{
Direkte Demokratie auf der transnationalen Ebene. Zur Entstehungsgeschichte der Europäischen Bürgerinitiative
}

\section{Bruno Kaufmann}

\section{Einleitung}

Deutschland ist bei weitem nicht das einzige Land Europas, in dem seit längerem die Möglichkeiten und Chancen der modernen direkten Demokratie als wesentlicher Bestandteil eines repräsentativen Systems heftig diskutiert werden. Es wird jedoch nicht nur diskutiert: Es gibt viele konkrete und praktische Reformansätze. So zum Beispiel in Schweden, wo die neue Verfassung - die seit Anfang 2011 gilt - das Recht auf lokale und regionale Bürgerinitiativen eingeführt hat ${ }^{1}$. Oder in Bulgarien, wo das Parlament sich Ende 2010 auf die Einführung einer ganzen Reihe von Initiativ- und Referendumsrechten auf allen politischen Ebenen einigen konnte. Die zentrale Frage, die immer mit dem Thema der modernen direkten Demokratie verbunden ist, geht über die reine Diskussion, $o b$ es ein Recht auf Bürgerinitiativen geben sollte oder nicht, hinaus. Die zentrale Frage dreht sich vielmehr um das Wie.

Im Falle der Europäischen Bürgerinitiative (EBI) wurde seit dem Jahre 2010 in der Tat vor allem um das Wie gerungen, nachdem Ende 2009 der Lissabonner Vertrag der Europäischen Union (EU) das neue Instrument eingeführt hat. Dieser Beitrag beleuchtet die fast zwei Jahrzehnte dauernde Entstehungsgeschichte des ersten transnationalen direktdemokratischen Volksrechtes der Geschichte und analysiert die intensiven Implementierungsanstrengungen, an denen sich neben offiziellen Akteuren auch zivilgesellschaftliche Organisationen sehr aktiv beteiligten. Ende 2010 konnten sich das Europäische Parlament und der Europäische Rat auf eine Verordnung zur Europäischen Bürgerinitiative einigen. Allerdings bleibt die konkrete Umsetzung in den Mitgliedsstaaten, was die Verifizierung von Unterschriften sowie die praktische Durchführung elektronischer Unterschriftensammlungen betrifft, offen. Ab Januar 2012 sollen Europäische Bürgerinitiativen lanciert werden können.

1 B. Kaufmann, Sweden: better late than never. A long way to a stronger initiative right in local politics, in: T. Schiller (Hrsg.), Direct Democracy in Local Politics in Europe (Direct Democracy in Modern Europe 2), 2. Aufl. 2011, i.E. 


\section{Die Geburtsstunde der Europäischen Bürgerinitiative}

\section{Brüssel, 12. Juni 2003}

Der 12. Juni 2003 war ein schöner, sonniger Tag. Nach dem nasskalten Frühling genoss man es in Brüssel, endlich wieder draußen in einem der vielen Cafés der Stadt zu sitzen. Auf der Place du Luxembourg, direkt vor dem Gebäude des Europäischen Parlaments, hatten sich die Mitglieder des „Konvents über die $\mathrm{Zu}$ kunft Europas“ - ein Ausschuss, der für den Entwurf einer ersten Verfassung der Europäischen Union gegründet worden war - zu einem Abschiedstrunk versammelt, da sich ihre 16-monatige Arbeit dem Ende zu neigte.

Im Parlament war jedoch das Präsidium des Konvents unter der Leitung des ehemaligen Präsidenten Frankreichs, Valéry Giscard d'Estaing, zu einer letzten formalen Sitzung zusammengekommen. Am folgenden Tag, dem 13. Juni, sollte die endgültige Fassung des Entwurfs dem Rat der Europäischen Union vorgelegt werden, der für nur eine Woche später ein Gipfeltreffen in Thessaloniki anberaumt hatte. Während die Sitzungen des Europäischen Konvents öffentlich gewesen waren, fand das Treffen des mächtigen Präsidiums, das sich nun über alle Vorschläge einigen musste, hinter verschlossenen Türen statt. Um 16:12 Uhr an diesem Donnerstagnachmittag gab die französische Nachrichtenagentur AFP zur großen Überraschung der vielen Beobachter eine Eilmeldung heraus: „In letzter Minute: Änderungen am Verfassungsvorschlag. [...] Es wird eine Europäische Bürgerinitiative eingeführt werden“. Drei Stunden später konnte Giscard d'Estaing diesen historischen Durchbruch bei einer Pressekonferenz im Parlament, wo der Konvent seit Februar 2002 gearbeitet hatte, bestätigen. Um 19:57 Uhr berichtete die Deutsche Presseagentur DPA: „Präsidium des Konvents bezieht eine Bürgerinitiative in den Verfassungsvorschlag mit ein. [...] Gesetzesvorschläge müssen jeweils von einer Million Bürgern aus mehreren Mitgliedsstaaten kommen.“2

Dieses war die offizielle Geburtsstunde der Europäischen Bürgerinitiative. Aber warum geschah dies gerade am 12. Juni 2003? Und wie konnte so eine Idee, die vor diesem schönen, sonnigen Tag in Brüssel als völlig utopisch angesehen worden war, zu einem Verfahren werden, das ab 2012 von fast einer halben Milliarde Menschen in Europa genutzt werden kann?

2 AFP, Convention: mises en garde contre des surenchères de dernière minute, in: B. Kaufmann/A. Lamassoure/J. Meyer (Hrsg.), Transnational Democracy in the Making. The New Challenge of European Initiative \& Referendum after the Convention, Amsterdam 2004, S. 244. 
Als Giscard d'Estaing, der selbsternannte „Gründungsvater“ des „Vertrags über eine Europäische Verfassung“, dieser grundlegenden Änderung hin zu modernen demokratischen Prinzipien auf grenzübergreifender Ebene zugestimmt hatte, waren viele Beobachter nicht nur überrascht, sondern auch höchst skeptisch gegenüber der praktischen Umsetzung der Beteiligung der Europäerinnen und Europäer. Die Überraschung vieler über dieses neue Instrument war wenig verwunderlich, denn sie wussten ja kaum etwas über die Geschichte jener Ideen, die zu diesem 12. Juni 2003 geführt hatten. Die Skepsis galt damals - zu Recht der Aussicht, den „Verfassungsvertrag“ je ratifizieren zu können. Heute aber stehen wir vor der Inkraftsetzung des ersten transnationalen und direktdemokratischen Instruments.

\section{Von Charles de Gaulle ...}

Bevor wir einen näheren Blick auf die Entstehung der Europäischen Bürgerinitiative werfen, sind ein paar Worte zur Beziehung zwischen moderner direkter Demokratie und europäischer Integration von Nöten. Am Anfang, als Europa noch darum rang, sich von der katastrophalen ersten Hälfte des 20. Jahrhunderts zu erholen - sowohl von zwei Weltkriegen als auch vom Nazismus und Stalinismus -, war es nicht klar, ob diese grenzübergreifende Versöhnung und Demokratisierung allein von den Eliten der Mitgliedsstaaten vorangetrieben werden sollte. Die erste gesamteuropäische Organisation, die nach dem Zweiten Weltkrieg gegründet wurde, der Europarat, war als Grundlage für eine wirklich grenzübergreifende Staatsorganisation in Europa gedacht und beruhte auf der Volkssouveränität der Bürgerinnen und Bürger. Eine Mischung aus dem elitären Widerwillen, eine direktere Form der Demokratie anzunehmen, der nordischbritischen Skepsis gegenüber dem europäischen Festland und der zunehmenden Kluft zwischen Ost und West - später durch den Bau der Berliner Mauer versinnbildlicht -, machte einen direkten Weg zu einer demokratischen Europäischen Union zunächst unmöglich. Ein Umweg von vier Jahrzehnten war nötig über Wirtschaftsabkommen, von oben veranlasste Staatsverträge und schließlich die Gründung eines Gemeinsamen Marktes in Westeuropa -, bevor mit dem Fall der Berliner Mauer im November 1989 die Schaffung einer demokratischen Europäischen Union in greifbare Nähe rückte.

Die Zeit, die benötigt wird, damit Ideen in die Praxis umgesetzt werden können, ist nicht immer gegeben. Aber so wichtige Ideen wie die direkte Beteiligung von Bürgerinnen und Bürgern an der Bestimmung der politischen Tagesordnung und an der Entscheidungsfindung verschwinden nicht so einfach, wenn sie erst 
einmal das Licht der Welt erblickt haben. Sie tauchen lediglich für eine gewisse Zeit ab. Bereits im Jahre 1949, als Gespräche um die Gründung einer Gemeinschaft für Kohle und Stahl begannen, verkündete der spätere französische Präsident Charles de Gaulle: ,... die Organisation Europas muss aus sich selbst hervorgehen. Ich denke, dass der Startschuss hierzu durch eine Volksabstimmung aller freien Europäer erfolgen sollte“. ${ }^{3}$

Eine Volksabstimmung über Europa als Gründungsakt eines politischen Europas! Das stellte sich auch der italienische Europaföderalist Altiero Spinelli vor, als er im Jahre 1964 die Schaffung einer EU-Verfassung vorschlug, die direkt von den Bürgerinnen und Bürgern ratifiziert werden sollte. ${ }^{4}$ Aber die Anhängerinnen und Anhänger von de Gaulles und Spinellis Ideen mussten geduldig abwarten, bis es an der Zeit für eine grenzübergreifendere direkte Demokratie war. Als klar wurde, dass die alte europäische Teilung, die die Demokratie im Westen in den Kühlschrank und im Osten in die Gefriertruhe verbannt hatte, vor dem Umbruch stand, wurden die ursprünglichen Vorschläge zur Einführung direktdemokratischer Elemente auf Europaebene wieder hervorgeholt. Im Jahre 1988 einigten sich das Europäische Parlament und andere EU-Einrichtungen auf Beschlüsse, die ,das Hinzuziehen von europäischen Bürgern zu Fragen über die EU“ ermöglichen sollten (JO C 187/231). Diese meist sehr vage formulierten Beschlüsse bezogen sich auf Ideen wie: „eine parallele Strategie, die dem Volk die Möglichkeit gibt, sich durch Volksinitiativen/Referenden zu äußern ... "55 und die Einführung von EU-weiten Konsultationen mit dem Volk/Meinungsumfragen. Im Dezember 1993 tat der europäische „Ausschuss für Bürgerliche Freiheiten, Justiz und Inneres (LIBE)“ seine Unterstützung für die Einführung eines „Referendums zur europäischen Gesetzgebung" ${ }^{\text {‘6 }}$ kund und stellte Bürgerabstimmungen über „Gemeinschaftsentscheidungen“7 in Aussicht.

Diese Impulse, die vom Europäischen Parlament ausgingen, sorgten dafür, dass im Vorfeld und während der Regierungskonferenz von Amsterdam Mitte der 1990er Jahre die mögliche Einführung eines formalen Rechts der EUBürgerinnen und -Bürger, eine Eingabe zu machen, zum ersten Mal diskutiert wurde. Ein weiterer, sehr konkreter Beitrag kam aus der Zivilgesellschaft: Eine der vielen Initiativen, die sich zu Zeiten des großen demokratischen und geopoli-

3 C. de Gaulle, Discours et messages. Dans l'attente. Février 1946-Avril 1958. Paris 1970, Bd. II, S. 309.

4 A. Spinelli, Una strategia per gli stati uniti d'Europa, Bologna 1989.

5 EP (1988). Resolution on ways of consulting European citizens about the EU. Brussels: JO C 187/231.

6 EP institutional commission. DOC A2-0332/88.

7 EP Commission. DOC A3-0031/94. 
tischen Wandels um 1990 hervorgetan hatten, war „eurotopia - das Netzwerk für transnationale direkte Demokratie in Europa“. Diese Gruppe von Menschen aus vielen verschiedenen Ländern Europas kam alle sechs Monate an einem anderen Ort in Europa zusammen, um die Vorschläge und Aussichten auf eine Europäische Verfassung mit direktdemokratischen Elementen zu diskutieren. Bis zum Juni 1994, als sich das Netzwerk in der norditalienischen Stadt Trient traf, hatte die Idee einer Europäischen Bürgerinitiative in verschiedenen europäischen sozialen Bewegungen schon eine erhebliche Eigendynamik entwickelt, so dass sie hier erstmals als öffentliche Idee vorgestellt werden konnte. Bei der Tagung in Trient waren auch Delegierte der Regierungen Italiens und Österreichs als Beobachter anwesend und erstatteten zu Hause über die Idee der Europäischen Bürgerinitiative Bericht. Das sorgte 1996-97 bei der Regierungskonferenz in Amsterdam für eine erste große Überraschung: Der damalige österreichische Außenminister Wolfgang Schüssel und sein italienischer Kollege Lamberto Dini stellten den ersten Entwurf für eine „Europäische Bürgerinitiative“ vor.

\section{3. ... zu Lamberto Dini}

Nach dem Schüssel-Dini-Vorschlag sollten zehn Prozent der Bürgerinnen und Bürger Europas (mit Unterschriften aus mindestens drei Ländern) das Recht haben, dem Europäischen Parlament eine Agendainitiative vorzulegen, die dann vom Parlament hätte in Betracht gezogen werden müssen. Dies war zwar eine schwache und - unter dem EU-Beschlusssystem - etwas fehlgeleitete Initiative mit viel zu hohen Hürden. Dennoch hatte die bloße Idee einer Bürgerinitiative nun die Aufmerksamkeit der „Vertragsherren“ - der Regierungen der Mitgliedsstaaten - gewonnen, die im Europäischen Rat versammelt waren.

Die Erfahrungen, die bei Regierungskonferenzen zur Vorbereitung und Entscheidung über EU-Vertragsänderungen gesammelt worden waren, brachten eine wichtige Erkenntnis mit sich: Grundlegende Reformen konnten nicht allein auf diese Weise erreicht werden. Nach den dramatischen Veränderungen in Europa im Jahre 1989 begannen nichtstaatliche Organisationen und akademische Kreise, mehr Interesse am Thema der transnationalen direkten Demokratie zu zeigen. Bei mehr als 20 Treffen innerhalb von zehn Jahren entwarf das Netzwerk „eurotopia“" sowohl Methoden zur Beteiligung der Bürgerinnen und Bürger an der Entwicklung einer transnationalen Verfassung als auch die ersten direktdemokratischen Elemente für eine solche Verfassung. Die Forderung nach einem Europäischen Konvent wurde schon Mitte der 1990er Jahre laut. Eine „doppelte qualifizierte Mehrheit“" wurde für das Gründungsreferendum über eine europäi- 
sche Verfassung vorgeschlagen: „Die Verfassung muss nicht nur von einer Mehrheit aller EU-Bürger angenommen werden, sondern auch von einer Mehrheit der Bürger in 4/5 der EU-Mitgliedsstaaten“. 8 Ab 1994, im Vorfeld der Regierungskonferenz von Amsterdam, bildeten eine Reihe von europäischen nichtstaatlichen Organisationen ein Netzwerk mit dem Namen „Inter Citizens Conferences“ (ICC): In der „Loccumer Erklärung“ wurden demokratische Voraussetzungen für eine Europäische Charta der Grundrechte festgelegt. Diese Erklärung beinhaltete auch zum ersten Mal den Vorschlag, beim Europäischen Parlament eine Initiative einzureichen. ${ }^{9}$

\section{Deutsche Aktivistenimpulse}

Gegen Ende der neunziger Jahre initiierte in Deutschland die Aktivistengruppe „Mehr Demokratie“ eine eigene Strategie für Europa: dabei wurde die Verfassungsidee zunächst gemieden und ein neues Netz gegründet: das „Network of Direct Democracy Initiatives in Europe“, das sich im Jahre 2002 in „Democracy International" umbenannte (und nun 2011 vorhat, sich in Form eines Vereins auch formell zu konstituieren). Es war diese Gruppe, die zusammen mit dem neu gegründeten „Initiative and Referendum Institute Europe“ die Ideen von „eurotopia“ für eine grenzübergreifende direkte Demokratie und für einen Europäischen Konvent am aktivsten vertrat. Nach der überraschenden Ablehnung des Vertrags von Nizza bei einer Volksabstimmung in Irland (6. Juni 2001) und wenige Tage nach dem europäischen Gipfeltreffen in Göteborg/Schweden (15./16. Juni 2001), das von gewaltsamen Protesten begleitet wurde, forderten Bürgerinnen und Bürger aus ganz Europa in Rostock abermals eine gemeinsame demokratische Zukunft: Sie verlangten einen Konvent für ein europäisches Verfassungsabkommen und schlugen die Einführung einer Europäischen Bürgerinitiative vor. Die darauffolgende Arbeit wurde vom „Rostocker Aufruf“ inspiriert, der am 17. Juni 2001 bei der 20. europäischen Versammlung von „eurotopia“ in Rostock verabschiedet worden war. Im ersten Jahrzehnt des neuen Jahrhunderts wurden aus den ,eurotopischen“ Ideen europäische Wirklichkeiten.

Zusammen mit dem Vorschlag zu einer Initiative von Dini/Schüssel im Jahre 1996 haben verschiedene nichtstaatliche Organisationen den Weg zu einer De-

8 The Rostock Process, 1991-2004: „On the way to more direct democracy in Europe“ (2001), S. 44.

9 R. Erne/A. Gross/B. Kaufmann/H. Kleger, Transnationale Demokratie - Impulse für ein demokratisch verfasstes Europa, 1995, S. $431 \mathrm{ff}$. 
batte über direktdemokratische Elemente in einer zukünftigen EU-Verfassung innerhalb und außerhalb des Konvents vorbereitet. ${ }^{10}$ Kurz nachdem der Konvent im Frühling 2002 zusammengekommen war, gründete das Initiative and Referendum Institute Europe ein „Convention Network“, das interessierte Mitglieder des Konvents und externe Expertinnen und Experten zusammenbrachte, um „die Bürger in den politischen Mittelpunkt zu rücken“. ${ }^{11}$ Dieses erste Treffen galt als Denkanstoß und brachte viele wichtige Mitglieder der ersten verfassungsgebenden Versammlung in der Geschichte der EU zusammen, so den späteren EBIRapporteur Alain Lamassoure (EPP-ED, Frankreich), Johannes Voggenhuber (Grüne/EFA-Österreich), Josep Borell Fontelles (PES-Spanien), Sylvia-Yvonne Kaufmann (GUE-Deutschland), Casper Einem (PES-Österreich) und Jürgen Meyer (PES-Deutschland). Jeder und jede von ihnen begann Ideen und Konzepte darüber zu entwickeln, wie direktdemokratische Elemente in die neuen EUVerträge mit einbezogen werden könnten. Am 20. Januar 2003 kam die Gruppe wieder zusammen, um ihre zahlreichen Vorschläge zu präsentieren und zu diskutieren, wie sowohl ein gesamteuropäisches Volksabstimmungsverfahren als auch eine Europäische Bürgerinitiative erreicht werden könnten. Anfang März 2003 stellte Lamassoure einen Entwurf vor: „Art. 43bis: droit de pétition et référendum européen“ (Europäisches Petitions- und Referendumsrecht). Dieser Vorschlag beinhaltete zwei Formen einer Europäischen Bürgerinitiative und die institutionelle Möglichkeit einer gesamteuropäischen Volksabstimmung über wichtige Sachthemen.

\section{Die Idee des Jürgen Meyer}

Nach einigen dramatischen Wochen und intensiver Lobbyarbeit von nichtstaatlichen Organisationen - der Aktivist Michael Efler von „Mehr Demokratie“ nann-

10 In Zusammenarbeit mit den Europaparlamentariern Heidi Hautala und Diana Wallis, sowie Michael Efler, Sprecher für europäische Angelegenheiten von „Mehr Demokratie“, entstand auf Vorschlag des Verfassers eine informelle Arbeitsgruppe zum Konvent. Mitglieder waren unter anderen Eduarda Azevedo, Péter Balázs, Michel Barnier, JensPeter Bonde, John Bruton, Panayiotis Demetriou, Karel De Gucht, Gijs De Vries, Lone Dybkjaer, Alexander Earl of Stockton, Casper Einem, Douglas Stewart, Joschka Fischer, Michael Frendo, Carlos Gonzalez Carnero, John Gormley, Sylvia-Yvonne Kaufmann, Alain Lamassoure, Jo Leinen, Linda Mc Avan, Iñigo Mendez de Vigo, Jürgen Meyer, Louis Michel, Alojz Peterle, Jacob Södermann.

11 B. Kaufmann/A. Lamassoure/J. Meyer (Hrsg.), Transnational Democracy in the Making, Amsterdam 2004, S. 223. 
te dies eine „Achterbahnfahrt in Richtung einer transnationalen Demokratie“"12 wurde schließlich der Vorschlag von Jürgen Meyer angenommen. Der Vorschlag (I-46, Teil I, Titel VI, CONV 724/03) ließ den Aspekt der Volksabstimmung völlig außen vor und konzentrierte sich voll und ganz auf die Bürgerinitiative. Meyers Schlüssel zum Erfolg war es, die Rolle der Bürgerinnen und Bürger in Bezug auf ihren Einfluss auf die Europäische Kommission mit den Initiativrechten des Parlaments und des Europäischen Rates gleichzusetzen. Letztendlich wurde der Vorschlag von 77 Mitgliedern $^{13}$ des Konvents unterzeichnet, was den letzten Widerstand im Präsidium des Konvents beseitigte und zu einem späten, aber willkommenen Durchbruch führte: Am letzten Sitzungsabend des Konvents wurde das Recht zur Bürgerinitiative in den Verfassungsentwurf mit einbezogen, was den Bürgerinnen und Bürgern zum ersten Mal in der Geschichte ein direktdemokratisches Instrument auf grenzübergreifender Ebene gab.

Mit der Aufnahme dieses zivilgesellschaftlichen Kernanliegens öffnete der Verfassungskonvent eine Tür für eine von unten kommende transnationale Beteiligung an der politischen Tagesordnung. Dieser letzte Entwurf eines Zusatzartikels schuf die Grundlage für den endgültigen Wortlaut der Verfassung, die am 13. Juni vom Vorsitzenden des Konvents, Giscard d'Estaing, vorgestellt wurde:

„Bürgerinitiative - Art. I-46, p 4

Mindestens eine Million Bürgerinnen und Bürger aus einer erheblichen Zahl von Mitgliedstaaten können die Kommission auffordern, geeignete Vorschläge zu Themen zu unterbreiten, zu denen es nach Ansicht der Bürgerinnen und Bürger eines Rechtsakts der Union bedarf, um die Verfassung umzusetzen. Die Bestimmungen über die besonderen

12 Ebda., S. 47.

13 Mitglieder: Akcam, Zekeriya; Amato, Guiliano; Andriukaitis, Vytenis; Athanasiu, Alexandru; Avgerinos, Paraskevas; Belohorska, Irena; Borrell Fontelles, Josep; Costa, Alberto Bernardes; Dam Kristensen, Henrik; De Rossa, Proinsias; Demetriou, Panayiotis; Dini, Lamberto; Duhamel, Oliver; Einem, Caspar; Fayot, Ben; Giannakou-Koutsikou, Marietta; Gricius, Algirdas; Haenel, Hubert; Helminger, Poul; Kaufmann, Sylvia-Yvonne; Kiljunen, Kimmo; Laborda, Gabriel Cisneros; Lequiller, Pierre; Marinho, Luis; Mavrou, Eleni; Oleksy, Jozef; Serracino-Inglott, Peter; Skaarup, Peter; Timermans, Frans; Vastagh, Pal; Voggenhuber, Johannes. Alternates: Abitbol, William; Alonso, Alejandro Munoz; Arabadjiev, Alexandar; Basile, Filadelfio Guido; Berger, Maria; Budak, Necdet; Carey, Pat; Carnero Gonzalez, Carlos; D'Oliveira Martins, Guilherme; Eckstein-Kovacs, Peter; Ene, Constantin; Floch, Jacques; Fogler, Marta; Garrido, Diego Lopez; Giberyen, Gaston; Gormley, John; Grabowska, Genowefa; Katiforis, Giorgos; Krasts, Guntars; Kroupa, Frantisek; Lichtenberger, Evelin; Mac Gormick, Neil; Maclennan of Rogart, Lord; Matsakis, Marios; Nagy, Marie; Nazare Pereira, Antonio; Severin, Adrian; Sivickas, Gintauta Speroni, Francesco; Spini, Valdo; Styllanides, Evripides; The Earl of Stockton, Alexander; Vassilou, Androula; Vella, George. Observers: Du Granrut, Claude; Sigmund, Anne-Marie; Sepi, Mario. 
Verfahren und Bedingungen, die für eine solche Bürgerinitiative gelten, werden durch ein Europäisches Gesetz festgelegt.“

Wie auch andere Elemente des Gesetzentwurfs (Arbeitsmethoden, Einbeziehung der Charta der Grundrechte, erhöhte Transparenz bezüglich der Tätigkeiten des Rats), symbolisiert die Europäische Bürgerinitiative eine Abwendung von der alten Europäischen Union mit nichtöffentlichen Debatten, Kuhhandel und engspurigen politischen Abwägungen.

Während sich der Europäische Konvent in seinem eigenen Verfassungsentwurf nicht auf einen Vorschlag zu einer gesamteuropäischen Volksabstimmung (Referendum) einigen konnte, begann die Aufnahme der Europäischen Bürgerinitiative in das neue Grundgesetz der Union eine immer größere Rolle zu spielen. Nachdem der „Vertrag über eine Verfassung für Europa“ am 29. Oktober 2004 durch die Staatsoberhäupter in Rom verabschiedet worden war, wurden in mehreren Mitgliedsstaaten - einer Mehrheit der EU-Staaten - Volksabstimmungen über den Vertrag angekündigt, so auch in Spanien, Frankreich, den Niederlanden, Luxemburg, Dänemark, Polen, Irland, dem Vereinigten Königreich, Portugal und Tschechien.

\section{Verlorene Ratifizierungsjahre - oder etwa nicht?}

\section{Direktdemokratische Dynamik}

Was juristisch unmöglich war und auch heute noch ist - eine unionsweite Volksabstimmung über ein Sachthema -, schien Anfang 2005 in Reichweite zu kommen. Die geplante Europäische Bürgerinitiative war im Vorfeld der spanischen Volksabstimmung am 20. Februar 2005 eines der Hauptargumente der Vertragsbefürworterinnen und -befürworter. Laut einer Meinungsumfrage des „Eurobarometers", die die Europäische Kommission nach der spanischen Abstimmung veröffentlichte, wussten $45 \%$ der Wahlberechtigten von der Existenz der neuen Europäischen Bürgerinitiative und $65 \%$ von ihnen glaubten, dass der Vertrag die Demokratie auf grenzübergreifender Ebene stärken würde. ${ }^{14}$ Am Ende stimmten $76,7 \%$ der teilnehmenden spanischen Wählerinnen und Wähler dem Vertrag zu.

Die direktdemokratische Dynamik, die durch den EU-Konvent und die anschließende Ja-Abstimmung in Spanien in Gang gesetzt wurde, veranlasste den Präsidenten der Europäischen Kommission, José Manuel Barroso, dazu, sich für

14 The European Constitution: Post-referendum survey; Eurobarometer 2005, S. 25, 28. 
mehr grenzübergreifende Demokratie stark zu machen. Nur ein paar Tage nach der spanischen Volksabstimmung hielt Barroso in Den Haag eine Rede, in der er erklärte, dass ,die Verfassung den Bürgerinnen und Bürgern neue Wege zur aktiven Beteiligung bei Entscheidungsfindungen gibt, indem sie eine Initiative vorbringen können, wenn diese von einer Million Unterschriften unterstützt wird“. Europas höchster Verwaltungschef fasste am Ende zusammen: „Vereinfacht ausgedrückt: Wir werden mehr Demokratie haben. “15

Diese erste offizielle Bewertung eines starken und direktdemokratischen Systems innerhalb der EU führte jedoch nicht zu einem leichtem Spiel und einer Ratifizierung des neuen Europäischen Vertrags im Schnellverfahren: In den folgenden beiden Volksabstimmungen in Frankreich (29. Mai 2005) und in den Niederlanden (1. Juni 2005) stimmte eine Mehrheit der Wählerinnen und Wähler gegen den Vertrag. Dies war gleichbedeutend mit einer Nicht-Ratifikation, da europäische Verträge von allen Mitgliedsstaaten angenommen werden müssen, um Unionsgesetz zu werden. Es bedurfte einer weiteren Runde an Verhandlungen und Volksabstimmungen (in Irland in den Jahren 2008 und 2009), bevor ein leicht abgeänderter Vertrag von Lissabon schließlich ratifiziert wurde und am 1. Dezember 2009 in Kraft treten konnte.

Während der „Römische“ Verfassungsvertrag nach der misslungenen Ratifizierung in mehr als einem Mitgliedsstaat juristisch erledigt war, gaben die Verfechter einer partizipatorischeren demokratischen Kultur in Europa nicht auf. Sie nutzten die folgenden Jahre für gründliche Überlegungen und Nachforschungen auf sub-europäischer (nationaler und regionaler) Ebene zu Erfahrungen mit Agendainitiativen, zogen daraus ihre Lehren und identifizierten die Hauptakteure für eine spätere Implementierung der Europäischen Bürgerinitiative.

\section{Das Instrument der Agendainitiative}

Zwischen 2005 und 2008 organisierte das Initiative and Referendum Institute Europe, das als Qualitätskontrolleur im Entstehungsprozess des ersten grenzübergreifenden und direktdemokratischen Instruments agierte, mehrere gesamteuropäische Beratungsrunden mit sowohl den staatlichen als auch den nichtstaatlichen Interessenvertreterinnen und -vertretern. Im Rahmen der Projekte zur „Initiative für Europa“" waren nichtstaatliche Organisationen dazu aufgefordert, bei J. Leinen/D. Wallis (Hrsg.), Initiative for Europe, Handbook 2008, 2007, S. 4 ff. 
Testläufen der Europäischen Bürgerinitiative zu helfen, noch bevor das neue Instrument überhaupt rechtlich implementiert war. Bei den jährlichen Gipfeltreffen zur Europäischen Bürgerinitiative wurden die Ergebnisse dieser Gutachten veröffentlicht und diskutiert. Einige nichtstaatliche Organisationen gewährten zusätzlich Einblicke in ihre Erfahrungen beim Ausprobieren dieses neuen Instruments. Die Erkenntnisse dieser Erwägungen und Beurteilungen wurden in der jährlichen Ausgabe des „Initiative for Europe“-Handbuchs veröffentlicht, herausgegeben von IRI Europe in Zusammenarbeit mit anderen demokratischen nichtstaatlichen Organisationen und den wichtigsten Parteien im Europäischen Parlament.

Und welche Haupterkenntnisse wurden aus dieser Arbeit gewonnen, die zwischen dem Scheitern des Verfassungsvertrags im Jahre 2005 und der Ratifizierung des Vertrags von Lissabon vier Jahre später stattfand? Drei Dinge sind dabei sehr deutlich geworden:

1) Die Erfahrungen mit agendabestimmenden Initiativrechten in Europa sind auf der nationalen Ebene nicht besonders ermutigend.

2) Die Bürgerinnen und Bürger Europas sind daran interessiert, so bald wie möglich grenzübergreifend an der politischen Tagesordnung teilzunehmen.

3) Es bedarf guter Sachkenntnis und großer Sorgfalt, um ein wirklich benutzerfreundliches und leistungsstarkes Format für die Europäische Bürgerinitiative zu entwickeln.

Im breiten Set der direktdemokratischen Volksrechte nimmt die Europäische Bürgerinitiative eine besondere Rolle ein. Vom Verfahren her steht die Agendainitiative per Definition zwischen einer Petition und einem Initiativvorschlag, welcher eine Volksabstimmung zu einem Sachthema nach sich zieht. Theoretisch und verfahrensmäßig haben die meisten Menschen im Großteil Europas eine Art von agendabestimmendem Instrument auf nationaler und/oder auf subnationaler Ebene. In 16 Ländern (zwölf von ihnen sind EU-Mitgliedsstaaten) ist der Vorgang auf nationaler Ebene bekannt. In weiteren acht Ländern (fünf EUMitgliedsstaaten) gibt es auf lokaler und/oder regionaler Ebene ein Recht auf Agendainitiativen.

\section{Eine spätere Kommissarin bricht das Eis}

Nach den negativ verlaufenen Volksabstimmungen zum Verfassungsvertrag im Jahre 2005 sah es für die Europäische Bürgerinitiative düster aus. Innerhalb der europäischen Institutionen, vor allem in der Europäischen Kommission, wurde jeder Versuch zur Schaffung einer Europäischen Bürgerinitiative ohne einen 
neuen Vertrag kategorisch abgeschmettert, während im Europäischen Parlament ähnliche Bestrebungen des Petitionsausschusses entschieden vom Ausschuss für konstitutionelle Fragen abgelehnt wurden.

Doch alleine schon die Idee, auch auf der grenzüberschreitenden Ebene initiativ werden zu können, regte ab Mitte des Jahrzehntes dutzende von zivilgesellschaftlichen Gruppen an, erste Test-EBIs zu lancieren. Ironischerweise kam die erste dieser Europäischen Bürgerinitiativen, die in den Medien für große Schlagzeilen gesorgt hat, aus dem Europäischen Parlament selbst: Cecilia Malmström, eine liberale Europaabgeordnete aus Göteborg in Schweden (wo sie 1995 an einer Versammlung von ,eurotopia“ teilgenommen hatte, in der das neue Instrument diskutiert worden war), gründete am Europatag 2006 die sogenannte „oneseat.eu“- (Ein [einziger] Sitz [des EU-Parlaments]) Initiative. Sie beschrieb ein sehr spezifisches und reales Problem und bot eine einfache Lösung an. Die Initiatoren bauten schnell ein Netz aus Personen (MdEPs aus allen Fraktionen im Parlament) und Organisationen (wie die Kampagne für eine parlamentarische Reform) auf. Mit dem Vorschlag, mehr Effizienz, Verantwortlichkeit und Transparenz zu zeigen, gelang es der ,oneseat“-Initiative das Thema auf die politische Tagesordnung zu setzen, obwohl es noch keine gesetzlich vereinbarte Grundlage gab, und ohne dass der Empfänger, die Europäische Kommission, zu diesem frühen Zeitpunkt direkt mit der Initiative konfrontiert wurde. Am 18. September 2006, einige Monate nachdem „oneseat“ ins Leben gerufen wurde, war es der junge Finne Olli Tikkanen, der als Millionster die Initiative unterzeichnete. Damit war bewiesen, dass es tatsächlich möglich sein würde, so viele Unterstützungserklärungen für eine Europäische Bürgerinitiative zu sammeln. Während sich die Webseite (www.oneseat.eu - noch immer aufrufbar) eindeutig auf die Vorkehrungen der EBI aus dem (aufgegebenen) Verfassungsvertrag bezog, brachte die „oneseat“-Initiative auch eine Reihe von Lehren für die Zukunft mit sich:

1. Die ,oneseat“-Initiative war eine reine Online-Initiative, und von daher mangelte es ihr an der nötigen und ergänzenden Informationsarbeit auf den Straßen Europas. Aus diesem Grund konnten nur wenige Länder ein großes Maß an Unterstützung generieren - wie zum Beispiel die Niederlande mit mehr als 430.000 und Schweden mit 140.000 Unterschriften.

2. Die ,oneseat“-Initiative lud absolut jede und jeden dazu ein, den Vorschlag (der zuweilen verwirrenderweise Petition genannt wurde) zu unterzeichnen, aber es gab kein internes oder technisches System, das die Identität der Unterzeichnenden sicherstellen konnte.

3. Außerdem sprach die ,oneseat“-Initiative ein Thema an, dass die EU-Kommission nicht von sich aus angehen kann. Der Sitz des Parlaments wird allein durch die EU-Mitgliedsstaaten entschieden. 
Diese drei Hauptdefizite führten dazu, dass der erste EBI-Pilotversuch schließlich in den Brüsseler Korridoren versandete. Als die Kommission sich weigerte, die Initiative auf Grund der Nichtzulässigkeit anzunehmen, wurden die Unterschriften an das Petitionskomitee des Parlaments weitergeleitet, aber die offizielle Antwort war wieder: „Nicht zulässig“ - dieses Mal, weil die Unterschriften nicht auf Echtheit überprüft werden konnten. Am Ende entschieden sich die Initiatoren mangels Partnern und organisatorischer Grundlagen gegen eine langfristige Planung. Ende September 2006 verließ Cecilia Malmström Brüssel und wurde EU-Ministerin der neuen konservativen Regierung in Schweden. Drei Jahre später kehrte sie nach Brüssel und ins Berlaymont-Gebäude zurück - nun als schwedisches Mitglied der EU-Kommission, wo ihr schon bald Europäische Bürgerinitiativen auf den Tisch kommen könnten.

\section{25 Testinitiativen}

Mit mehr als zwei Dutzend Europäischen Bürgerinitiativen in Pilotversuchen wurde das neue Instrument ausgiebig, in Echtzeit und in großem Maßstab erprobt. Diese Anwendungen haben gezeigt - wenig überraschend -, dass das Instrument für alle möglichen Akteure und Interessenvertreter von Nutzen sein kann, einschließlich politischer Parteien und großer Organisationen wie Gewerkschaften oder Greenpeace, aber auch für engagierte Einzelpersonen und Ad-hocGruppen. Besonders letztere haben versucht, den Vorgang mit dem Ziel der Demokratisierung der europäischen Demokratie zu nutzen: Gleich mehrere EBIs wurden ins Leben gerufen, um eine gesamteuropäische Volksabstimmung über den neuen europäische Grundlagenvertrag auszulösen. Eine weitere Schlüsselinitiative hieß einfach „die EBI-Initiative“ oder „die Initiative für die Initiative“. Mit Unterstützung des Aktivistennetzes Democracy International und des Europäischen Studentenforums hat Ende 2006 eine Gruppe junger Europäerinnen und Europäer die „EBI-Initiative“ gegründet, als nach dem Scheitern des Verfassungsvertrags klar wurde, dass es noch eine Zeit lang bis zur Zustimmung und Ratifizierung eines neuen EU-Vertrags dauern würde (was Ende 2009 geschah). Sie erhielten Unterstützung von vielen anderen Organisationen - wie zum Beispiel vom European Citizen Action Service und der König-Baudouin-Stiftung -, aber auch von vielen Mitgliedern des Europäischen Parlaments. Die Umstände für diese Europäische Bürgerinitiative waren jedoch nicht einfach, da nicht nur noch keine formale Verordnung für eine EBI existierte, sondern auch das Hauptziel, die Forderung einer formalen Einführung des Rechts zur Europäischen Bürgerinitiative ohne einen neuen EU-Vertrag, nicht wirklich von den Hauptinstitu- 
tionen der EU unterstützt wurde. Während die Initiative sehr erfolgreich darin war, die Vorbereitungsarbeit für die Implementierung der Europäischen Bürgerinitiative auf allen Ebenen innerhalb der unterstützenden Organisationen und in den EU-Institutionen aktiv am Leben zu halten, scheiterte sie daran, eine ausreichende Anzahl an offiziellen Unterschriften in Europa zu sammeln.

Die ersten 25 Pilotinitiativen ${ }^{16}$ können auch als Vorschau auf die Art und Weise gesehen werden, wie die Europäische Bürgerinitiative von vielen verschiedenen Gruppen mit unterschiedlichen politischen Hintergründen und für viele verschiedene Zwecke genutzt werden wird. Auch Gemeinsamkeiten lassen sich finden: der intensive Gebrauch elektronischer Hilfsmittel, die notwendige Mehrsprachigkeit sowie ein wachsendes Verständnis dafür, dass die Europäische Bürgerinitiative ein langwieriger Prozess ist und dass sie nur ein ergänzender Aspekt eines Langzeitprojektes sein kann. Basierend auf diesen Erfahrungen können wir davon ausgehen, dass die Europäische Bürgerinitiative vorwiegend proaktiv und weniger reaktiv verwendet werden wird. Warum? Weil sich dieses Instrument nicht gut für emotionsgeladene (Re)aktionen, sondern eher für langfristig angelegte agendabestimmende und anwendungsbezogene Vorhaben eignen dürfte.

\section{Die Fragen der Verfahrensausgestaltung}

\section{Enger Terminkalender}

Nach dem Inkrafttreten des Lissabonner Vertrages gelangte die Europäische Bürgerinitiative (Art. 11. Abs. 4 des Vertrages) in die gesetzliche Implementierungsphase. Folgende Termine und Akteure standen dabei im Mittelpunkt:

16 Eine detaillierte Übersicht und Auflistung zu sämtlichen Test-EBIs findet sich im „Handbuch zur Europäischen Bürgerinitiative - eine Gebrauchsanweisung zur transnationalen, direkten Demokratie“, Green European Foundation, Brüssel 2011. 


\begin{tabular}{|c|c|c|}
\hline Wann? & Wo? & Was? \\
\hline 7. Mai 2009 & $\begin{array}{l}\text { Europäisches } \\
\text { Parlament }\end{array}$ & $\begin{array}{l}\text { Beschluss über die Implementierung der Bürgerini- } \\
\text { tiative }^{17}\end{array}$ \\
\hline 9. Mai 2009 & $\begin{array}{l}\text { Gipfeltreffen zur } \\
\text { Europäischen Bürger- } \\
\text { initiative }^{18}\end{array}$ & $\begin{array}{l}\text { Das Salzburger Manifest für die Europäische } \\
\text { Bürgerinitiative, } 2009^{19}\end{array}$ \\
\hline $\begin{array}{l}\text { 11. Novbr. } \\
2009\end{array}$ & $\begin{array}{l}\text { Europäische } \\
\text { Kommission }\end{array}$ & Grünbuch über eine Europäische Bürgerinitiative ${ }^{20}$ \\
\hline $\begin{array}{l}\text { 11. Novbr. } \\
2009-31 \\
\text { Januar } 2010\end{array}$ & Öffentliche Anhörung & $\begin{array}{l}323 \text { Beiträge: } 159 \text { Bürger, } 133 \text { nichtstaatliche } \\
\text { Organisationen, } 31 \text { Regierungsorganisationen }{ }^{21}\end{array}$ \\
\hline 17. März 2010 & $\begin{array}{l}\text { Europäischer } \\
\text { Wirtschafts- und } \\
\text { Sozialausschuss }\end{array}$ & $\begin{array}{l}\text { Stellungnahme zur Implementierung des Vertrags von } \\
\text { Lissabon: partizipatorische Demokratie und die } \\
\text { Bürgerinitiative }^{22}\end{array}$ \\
\hline 31. März 2010 & $\begin{array}{l}\text { Europäische } \\
\text { Kommission }\end{array}$ & Verordnungsentwurf für die Bürgerinitiative ${ }^{23}$ \\
\hline 9. Mai 2010 & $\begin{array}{l}\text { Gipfeltreffen zur } \\
\text { Europäischen Bürger- } \\
\text { initiative }\end{array}$ & $\begin{array}{l}\text { Salzburger Manifest für eine direkte Demokratie in } \\
\text { Europa, } 2010\end{array}$ \\
\hline Juni 2010 & $\begin{array}{l}\text { Ausschuss der } \\
\text { Regionen }\end{array}$ & $\begin{array}{l}\text { Stellungnahme zum Verordnungsentwurf für die } \\
\text { Europäische Bürgerinitiative }\end{array}$ \\
\hline 14. Juni 2010 & Europäischer Rat & $\begin{array}{l}\text { Generelle Sichtweise des Rats für Allgemeine } \\
\text { Angelegenheiten }^{24}\end{array}$ \\
\hline
\end{tabular}

17 http://www.europarl.europa.eu/sides/getDoc.do?pubRef=-//EP//TEXT+TA+P6-TA-20090389+0+DOC+XML+V0//EN.

18 Seit 2007 organisieren Nichtregierungsorganisationen, die von IRI Europe koordiniert werden, jährliche Treffen, um die Entwicklung der EBI zu beobachten und fördern. Seit 2009 werden diese Treffen rund um den Europatag in Salzburg/Österreich abgehalten: www.legalpolicy.eu.

19 The European Citizens' Initiatives, 2010, S. 119 ff.

20 http://ec.europa.eu/dgs/secretariat_general/citizens_initiative/docs/com_2009_622_ en.pdf.

$21 \mathrm{http} / / /$ ec.europa.eu/dgs/secretariat_general/citizens_initiative/contributions_en.htm.

22 http://eescopinions.eesc.europa.eu/EESCopinionDocument.aspx?identifier=ces $\backslash$ souscomitelsc032\%20la $\% 20$ mise $\% 20$ en $\% 20$ oeuvre $\% 20 \mathrm{du} \% 20$ traite $\% 20 \mathrm{de} \% 20$ lisbonnelces 4 65-2010_ac.doc\&language $=\mathrm{EN}$.

23 http://ec.europa.eu/dgs/secretariat_general/citizens_initiative/docs/com_2010_119_ en.pdf.

24 2010/0074 (COD). 


\begin{tabular}{l|ll}
\hline 14. Juli 2010 & $\begin{array}{l}\text { Europäischer } \\
\text { Wirtschafts- und } \\
\text { Sozialausschuss }\end{array}$ & $\begin{array}{l}\text { Vorschlag für eine Regulierung der Bürgerinitiative } \\
\text { durch das Europäische Parlament und den Rat }{ }^{25}\end{array}$ \\
$\begin{array}{l}\text { 30. Novbr. } \\
\text { 2010 }\end{array}$ & $\begin{array}{l}\text { Europäisches } \\
\text { Parlament (Komitees) }\end{array}$ & $\begin{array}{l}\text { Abstimmung über den Bericht im Ausschuss für } \\
\text { konstitutionelle Fragen (AFCO) und im } \\
\text { Petitionsausschuss (PETI) }\end{array}$ \\
$\begin{array}{l}\text { 16. Dezember } \\
2010\end{array}$ & $\begin{array}{l}\text { Europäisches } \\
\text { Parlament }\end{array}$ & $\begin{array}{l}\text { Schlussabstimmung (628 zu 15 Stimmen) im Parlament } \\
\text { nach Einigung mit dem Rat }\end{array}$ \\
\hline $\mathbf{2 0 1 2}$ & Europäische Union & $\begin{array}{l}\text { Inkrafttreten der Verordnung und Vorbereitung des } \\
\text { Verfahrens in den Mitgliedstaaten }\end{array}$ \\
\hline
\end{tabular}

Nach vielen Jahren der Diskussion über die Prinzipien der modernen repräsentativen Demokratie des 21. Jahrhunderts auf grenzübergreifender Ebene - gleichermaßen basierend auf der direkten und der indirekten Demokratie - und nach Jahren der praktischen Vorbereitung der Bürgerinnen und Bürger Europas machte der letzte verfahrenmäßige Feinschliff am Instrument deutlich, welches Potential die Sache hat: Zum ersten Mal in der Geschichte beschäftigten sich staatliche und nichtstaatliche Organisationen aus ganz Europa mit der gleichen Demokratie-Frage: Wie kann so ein direktdemokratisches Instrument am besten aufgebaut werden? Dieser Vorgang schloss alle wichtigen europäischen Institutionen wie den Europäischen Rat, das Parlament und natürlich die Kommission mit ein, aber auch andere, wie zum Beispiel den Europäischen Wirtschafts- und Sozialausschuss (EWSA), den Ausschuss der Regionen und den Europäischen Bürgerbeauftragten.

Während alle die direktdemokratische Aufwertung auf transnationaler Ebene willkommen hießen, sehen sich die Institutionen interessanterweise dabei samt und sonders in einer eigenen wichtigen Rolle - so möchte zum Beispiel das Parlament den EBI-Prozess überwachen, und der EWSA möchte sich als Anlaufstelle für interessierte europäische Bürgerinnen und Bürger und Initiativgruppen zur Verfügung stellen. Wenn wir kurz auf das jahrhundertelang währende Ringen um die beste Form der repräsentativen Demokratie zurückblicken, ist diese neue Einstellung und Anerkennung einer modernen repräsentativen Demokratie, die indirekte (parlamentarische) und direkte (partizipatorische) Verfahren der

$25 \mathrm{http} / /$ www.eesc.europa.eu/?i=portal.en.institutional-reform-opinions-resolutions-opinions. 10571 . 
Agendabestimmung und Entscheidungsfindung miteinander verbindet, ein wirklich globaler Durchbruch. Es öffnet Türen und schafft neue Energien für die praktische Umsetzung einer direkten Demokratie in Europa (und über Europas Grenzen hinaus). Dies hat den Vizepräsidenten der Europäischen Kommission, Marô̂ Sefĉovich, zu folgender Aussage veranlasst:

„,... die Bürger wollen, dass dieses Instrument benutzerfreundlich wird. Sie wollen, dass es einfach, unkompliziert, verständlich und vor allem leicht anwendbar ist! Ich sehe das ganz genau so. Dieses Instrument muss genutzt werden. Wir müssen es so benutzerfreundlich wie möglich gestalten, damit es den öffentlichen Raum in Europa unterstützt, den Bereich für öffentliche Debatten in Europa erweitert und die EU näher an die Belange der Bürger rückt. “26

Eine recht beeindruckende und allumfassende Aussage, geradezu ein Appell, eines hohen Vertreters der Institution, die Hauptansprechpartnerin der kommenden Europäischen Bürgerinitiativen sein wird. Dieses Statement ist beinahe wie eine Kontrollliste aller Dinge, die die Europäische Kommission bei der Europäischen Bürgerinitiative bedenken sollte.

\section{Positive Einstellung, schwierige Details}

Im Vorfeld der Präsentation des Verordnungsentwurfs der Kommission am 31. März 2010 hatten im Grunde alle teilnehmenden Interessenvertretungen diese positive Einstellung sowohl gegenüber der Einführung der Europäischen Bürgerinitiative als auch über die Einrichtung eines bürgerfreundlichen, anwendbaren und einfachen Vorgangs vertreten, was dieses erste direktdemokratische Instrument auf grenzübergreifender Ebene zum Erfolg führen könnte. Interessanterweise waren selbst Regierungen von Mitgliedstaaten wie Schweden, Tschechien, Deutschland und Griechenland, wo es keine vergleichbaren Möglichkeiten auf nationaler Ebene gibt, klar für die Einführung der Europäischen Bürgerinitiative. Ferner nahmen viele regionale Regierungen und nationale Parlamente am Konsultationsprozess teil, was jüngste Entwicklungen zur Stärkung direktdemokratischer Elemente auf diesen Ebenen in Ländern wie zum Beispiel Italien, Spanien und Deutschland widerspiegelt.

26 Rede von Maroš Šefčovič, Vizepräsident der Europäischen Kommission, verantwortlich für „Zwischenbehördliche Beziehungen und Administration“, bei der Anhörung der Interessenvertreter der Europäischen Bürgerinitiative am 22. Februar 2010: http://ec.europa.eu/dgs/secretariat_general/citizens_initiative/stakeholder_hearing_en.htm. 
Daraus kann abgeleitet werden, dass ein allgemeines positives Interesse daran besteht, die repräsentative Demokratie repräsentativer zu gestalten, und dass ein weit verzweigtes Netz aus staatlichen und nichtstaatlichen Interessenvertretern bereit ist, diesen neuen Prozess zu fördern, zu unterstützen und zu überwachen, sobald er 2011 beginnt.

Im Bezug auf die verfahrensrechtliche Einstellung gegenüber der Verordnung hat die vor-gesetzgebende/konsultative Phase der Entwicklung der Verordnung (bis zur Präsentation des Vorschlags der Kommission am 31. März) gezeigt, dass die meisten Interessenvertretungen - zusätzlich zu ihrer allgemeinen positiven Einstellung - stark von eigenen Erfahrungen ausgehen und zum Beispiel subnationale Erfahrungen bei der Sammlung von Unterschiften oder bei der Rückerstattung von Finanzmitteln in den Mittelpunkt stellen. Das Spektrum an verschiedenen Kommentaren hat ebenfalls gezeigt, dass einige Interessenvertretungen die Europäische Bürgerinitiative weiterhin eher als traditionellen Petitionsprozess betrachten, während andere das neue Instrument als volle klassische Bürgerinitiative mit Wirkung auf Entscheidungsfindungen empfinden (wollen).

Der Vorschlag der Kommission, mit dem der offizielle rechtliche Prozess der Gesetzgebung gestartet wurde, verbindet eine Reihe von großzügigen und einschränkenden Elementen.

Die großzügige Seite des Vorschlags:

- er wählt eine freie Form der Unterschriftensammlung, einschließlich - als Neuheit - der Online-Sammlung von Unterstützungserklärungen (Art. 5 und 6);

- er heißt ein Registrierungsformat willkommen, das als administrativer Schritt zu verstehen ist und die Zulässigkeitsprüfung oder politische Einstufung nachordnet (Art. 4);

- er erlaubt Mitgliedsstaaten, die Unterschriften stichprobenartig zu überprüfen (Art. 9).

Jedoch steht klar auf der einschränkenden Seite des Vorschlags:

- die hohe Zahl von neun Mitgliedsstaaten, die für die Sammlung von Unterschriften als Minimum vorausgesetzt wird. Mit dieser Bedingung steht die Kommission allein auf weiter Flur, da während des konsultativen und legislativen Prozesses die meisten Interessenvertretungen eine kleinere Zahl an Staaten vorgeschlagen hatten, aus denen die Unterschriften kommen müssen;

- die extrem hohe Zahl an Unterschriften (300.000), die von den Organisatoren gesammelt werden müssen, bevor eine Zulässigkeitsprüfung durchgeführt werden kann; und 
- die Voraussetzungen, die im Zusammenhang mit dem sogenannten „Formular für eine Unterstützungsbekundung“ (Anhang III) stehen, das nicht nur den Namen, die Adresse, die Staatsangehörigkeit und das Geburtsdatum des Unterzeichnenden verlangt, sondern auch eine Reihe von Ausweisummern - diese Daten sind nicht nur unterschiedlich von Land zu Land, sie sind auch in einigen Mitgliedsstaaten explizit nicht vorhanden.

Auf einer weiteren Ebene liefert der Vorschlag der Kommission einige sehr konstruktive und nützliche Beiträge, während andere Aspekte komplett ignoriert oder völlig falsch eingeschätzt werden.

Die konstruktiven Elemente umfassen:

- die Einbeziehung einer Reihe von Formularen, die den Vorgang wesentlich verlässlicher, überprüfbarer und transparenter gestalten wird - beispielsweise das „Formular für eine Unterstützungsbekundung“ (Anhang III), das „Formular für die Einreichung von Interessenbekundungen“ (Anhang VI) und die „Bescheinigung der Zahl der gesammelten gültigen Unterstützungserklärungen“" (Anhang VII), was die Anlieferung von Stapeln von Unterschriftsformularen nach Brüssel unnötig macht;

- der Vorschlag einer festgelegten Mindestzahl an gültigen Unterschriften für jeden Mitgliedsstaat (Anhang I), gestuft in Abhängigkeit der Sitze des jeweiligen Landes im Europäischen Parlament. Dieses erleichtert es bevölkerungsreichen Mitgliedsstaaten, sich zu qualifizieren;

- die berechtigterweise vorsichtigen und sorgfältigen Vorbereitungen für das anvisierte elektronische Sammlungssystem, das Schritt für Schritt im Einklang mit nationalen Behörden im Laufe der ersten Jahre der Anwendung eingeführt werden muss (Art. 19). Dies führt zur Einbindung einer Überprüfungsklausel (Art. 21), die den ganzen Prozess fünf Jahre nach Inkrafttreten der Verordnung zurück an die Legislative und die europäische Öffentlichkeit tragen wird.

Während die großzügigen und einschränkenden Elemente des Verordnungsentwurfs ausführlich von allen Interessenvertretungen diskutiert wurden und werden, haben die konstruktiven Aspekte von beinahe allen, die Feedback gegeben haben, Zuspruch erhalten. Trotzdem hat die Kommission einige andere Aspekte ignoriert und/oder unterschätzt:

- die administrative und politische Sicherheitsfunktion der ersten Schritte (Beratungsdienste besonders bei der Registrierung). Tatsächlich sieht der Verordnungsentwurf nur einen (1) Beamten und einen (1) Assistenten für die Bearbeitung aller verwandten Aufgaben vor - ein wahrlich unterbesetztes Büro (was noch reichlich untertrieben ist!); 
- die Rolle der finanziellen Unterstützung für Organisationen, die weder starke Organisationen noch etablierte Interessen vertreten. Gleichzeitig schlägt der Verordnungsentwurf vor, finanzielle Transparenz aller Organisationen zu verlangen, von denen erwartet wird, dass sie zum Zeitpunkt der Registrierung alle Quellen zur Finanzierung und Unterstützung der geplanten Initiative offenlegen (Anhang II-7); und

- nicht zuletzt der offensichtliche Widerwille, eine konkrete Verpflichtung zu einer Reaktion von Seiten der Kommission auf eine Europäische Bürgerinitiative, die alle erwähnten Voraussetzungen erfüllt hat, mit einzubeziehen (Art. 11-1-ab). Dieses steht in starkem Widerspruch zu den Versprechungen, die das Kommissionsmitglied Sefĉoviĉ beim oben zitierten Treffen mit Interessenvertretungen am 22. Februar 2010 verkündet hatte. $^{27}$

Zusammenfassend lässt sich sagen, dass der Verordnungsentwurf der Kommission, der den formalen gesetzgebenden Prozess ins Rollen gebracht hat, auf folgenden Punkten basierte:

- $\quad$ einem vernünftigen und angemessenen Grundsatz, wie von Vizepräsident Sefĉovic formuliert: benutzerfreundlich, einfach, unkompliziert, verständlich, leicht anwendbar;

- einer Mischung aus großzügigen und einschränkenden Regulierungsvorschlägen zusammen mit mehreren konstruktiven Elementen und Instrumenten, während andere beinahe völlig umgangen wurden.

3. Wirtschafts- und Sozialausschuss

Im Zusammenhang mit dem formalen gesetzgebenden Prozess, der mit der Präsentation des Verordnungsentwurfs der Kommission am 31. März 2010 begann, wurde ein Hauptaugenmerk auf mögliche Verbesserungen, Klarstellungen und Veränderungen des EBI-Gesetzes gelegt.

Dazu gehörten:

- die Äußerung des Europäischen Rats vom 14. Juni 2010, welche im Wesentlichen den Verordnungsentwurf der Kommission bestätigt - besonders die sperrige Forderung nach Unterzeichnenden aus zumindest einem Drittel der Mitgliedsstaaten bei der Unterschriftensammlung - und vorschlägt, die für

27 Beim Kommissionstreffen sagte Ŝefçoviĉ, dass „erfolgreiche Bürgerinitiativen die versammelte Kommission zu einer ernsthaften Berücksichtigung der Forderungen von Millionen von Bürgern bewegen" werden. 
die Zulässigkeitsprüfung benötigten 300.000 Unterschriften auf 100.000 herunterzusetzen. Zudem ist der Rat konkreter in seinen Aussagen zu Themen wie der Onlinesammlung von Unterschriften, dem Datenschutz und den Identifizierungsanforderungen für die Unterstützungserklärungen. Er schlägt vor, dass letztere Anforderung aufgrund der riesigen Vielfalt von berechtigten Ausweisformen in Europa ganz und gar vom endgültigen Gesetz gestrichen werden sollte;

- der Ausschuss der Regionen schlug vor, die Anzahl der „qualifizierten“ Mitgliedsstaaten auf ein Viertel der Gesamtzahl herabzusetzen. Dies rechtfertigt er durch einen Vergleich mit verwandten Abkommensregelungen zur Agendabestimmung, wie zum Beispiel Art. 76 EUV. In anderer Hinsicht jedoch verkomplizieren viele Vorschläge des AdR den Vorgang eher, als dass sie ihn erleichtern - wie zum Beispiel weitere Ausschlussmöglichkeiten im Bezug auf die Eignung einer Initiative bei der Registrierung, der Zusammenschluss der Registrierung und der Zulässigkeitsprüfung und eine zusätzliche Forderung, die den Organisatoren die Veröffentlichung einer Liste aller geplanten Ausgaben vorschreibt. Am sonderbarsten von allen - aber offensichtlich aus der Idee eines neuen Instruments entsprungen - war der Vorschlag des AdR, dass auch Regionen dazu berechtigt sein sollten, ihre Europäische Bürgerinitiative zu organisieren;

- die Meinung des Europäischen Wirtschafts- und Sozialausschusses, der seine eigenen Ansichten in einem breitgefächerten Zwei-Phasen-Prozess geformt hatte, zeigte zunächst die umfassenden Möglichkeiten und Grenzen der Europäischen Bürgerinitiative auf (im März von der Versammlung des EWSA angenommen) und bot dann eine Liste mit Verbesserungsvorschlägen, einschließlich einer Zulässigkeitsprüfung bei 50.000 Unterschriften (im Zusammenhang mit einem Übersetzungsangebot) und eine voll entwickelte Idee für eine unterstützende Infrastruktur für die Europäische Bürgerinitiative.

4. Die europäische Sieben - ein weiterer Meilenstein

Schliesslich gelang es dem Europäischen Parlament und dem Rat sich Ende 2010 auf eine Verordnung zu einigen, welche das Verfahren auf den ersten Blickrecht praktikabel erscheinen lässt. Dazu gehört, dass

- es nunmehr nicht mehr 300.000 Unterschriften braucht, wie von der Kommission ursprünglich vorgeschlagen, bevor sich die Behörden mit der Zulässigkeit einer Europäischen Bürgerinitiative beschäftigen; 
- stattdessen für die Registrierung (und gleichzeitig formale Zulässigkeitsprüfung) die Bildung eines mindestens siebenköpfigen Iniativkomittees erforderlich ist, dessen Mitglieder aus wiederum mindestens sieben Mitgliedsstaaten kommen;

- für die initiale Prüfung der Kommission maximal zwei Monate Zeit zur Verfügung stehen. Zudem können die Initiantinnen und Initiatianten gegen einen allfälligen negativen Entscheid bei Europäischen Gerichten rekurrieren;

- der Zeitraum der Unterschriftensammlung maximal zwölf Monate in Anspruch nimmt und dass die erforderliche eine Million Unterschriften aus mindestens sieben qualifizierten Mitgliedsstaaten kommen;

- den Bürgerinnen und Bürgern seitens der EU bei der Vorbereitung und Durchführung einer Initiative ein „Help Desk“ zur Seite gestellt werden soll, wobei Umfang und Struktur dieser Dienstleistung noch nicht ganz klar sind.

Ingesamt ist es also im Verlaufe der Behandlung des Kommissionsvorschlages gelungen, diesen bürger- und demokratiefreundlicher zu machen. In mindestens zwei Beziehungen bleibt die Verordnung, die im Frühjahr 2011 in Kraft tritt, aber noch so unklar, dass noch keine wirklichen Prognosen über die künftige Praktikabilität des ersten transnationalen Volksrechtes der Geschichte abgegeben werden können. Dies betrifft,

- die Frage der Verifizierbarkeit von Unterstützungsunterschriften - im neuen EU-Jargon „Unterstützungserklärungen“ genannt - ist abschliessend von den einzelnen Mitgliedsstaaten zu klären, die damit unterschiedlich umgehen können bzw. auch zusätzliche Identifikationsinformationen (wie z.B. Passnummern) von Unterstützerinnen und Unterstützern verlangen; hier besteht die Gefahr, dass es unterschiedlich schwierig sein wird, in den verschiedenen Mitgliedsstaaten für eine Initiative zu sammeln;

- die innovative Möglichkeit, für eine Volksinitiative auch auf dem elektronischen Weg Unterschriften zu sammeln. Einerseits muss hierzu erst noch eine gesamteuropäische Spezifikation festgelegt werden, aufgrund derer dann die Software bzw. die Softwares entwickelt und von jedem Mitgliedsstaat einzeln zertifiziert werden. Das schafft zusätzlichen (negativen) Spielraum zur Erschwerung des Initiativprozesses. Zudem bleibt unklar, wie ohne gesamteuropäische Erfassung der Unterschriften Mehrfachunterstützer identifiziert und Auslandbürger einem Mitgliedsstaat zugeordnet werden können.

Die Einigung über die Verordnung im Dezember 2010 war ein weiterer Meilenstein in der Entstehungsgeschichte der Europäische Bürgerinitiative - aber nicht der letzte, bevor im Frühjahr 2012 (hoffentlich) die ersten Europäischen Bürgerinitiativen lanciert werden können. 